\title{
Revista Brasileira de Enfermagem REBEn \\ Incidência e características de Cesáreas e de partos normais: estudo em uma cidade no interior do Ceará
}

\author{
Incidence and features of Cesarean Section and natural childbith: \\ study in a city from Ceará's countryside \\ Incidencia y características de Cesáreas y partos normales: \\ estudio en una ciudad del interior de Ceará
}

\section{Maria Veraci Oliveira Queiroz}

Enfermeira do Hospital Geral de Fortaleza. Mestre e Doutora pelo Programa de PósGraduação em Enfermagem da Universidade Federal do Ceará. Docente da Universidade Estadual do Ceará (UECE). Vice-Coordenadora do Curso Mestrado Acadêmico em Cuidados Clínicos. Participante do grupo de Pesquisa Saúde Mental, Família e Práticas de Saúde da

UECE.

veraciog@hotmail.com

\section{Nara Suelene Jacobina e Silva}

Enfermeira do Hospital de Baturité (Ceará). Especialista em Enfermagem Obstétrica pela Universidade Estadual do Ceará.

Maria Salete Bessa Jorge

Enfermeira. Docente da Universidade Estadual do Ceará. Doutora em Enfermagem pela EERP/ USP. Titular em Enfermagem em Saúde Mental. Presidente do Comitê de ètica em Pesquisa da UECE. Pesquisadora do CNPq. Coordenadora do Curso de Mestrado Acadêmico em Saúde

Pública. Líder do grupo de Pesquisa Saúde Mental, Família e Práticas de Saúde da UECE. masabejo@hotmail.com

Thereza Maria Magalhães Moreira

Enfermeira. Mestre e Doutora pelo Programa de Pós-Graduação em Enfermagem pela Universidade Federal do Ceará. Docente da Universidade Estadual do Ceará (UECE). Coordenadora do Curso Mestrado Acadêmico em Cuidados Clínicos. tmmmoreira@yahoo.com.br

\section{RESUMO}

A pesquisa objetivou descrever as características da população estudada, como estado civil, ocupação e número de consultas de pré-natal; verificar a incidência de cesárea e de parto normal em clientes atendidas em uma instituição; comparar essas incidências no período pesquisado, discutindo as indicações de cesarianas mais freqüentemente referidas; Estudo descritivo retrospectivo baseado em prontuários de uma instituição filantrópica conveniada ao Sistema Único de Saúde. Evidenciou-se um elevado percentual de parto cesareano com alta incidência em adolescentes. Constatou-se que as principais indicações de cesárea foram ter história de cesárea anterior e doença hipertensiva específica da gravidez. Registrou-se predomínio de gestantes com 4 a 7 consultas de prénatal. Mudanças na assistência pré-natal podem ter impacto na taxa de cesárea, preparando a mãe para 0 trabalho de parto, aspecto intimamente relacionado à capacitação de parteiras, enfermeiras obstetras e médicos, como também substituição da assistência individual por uma assistência de equipe.

Descritores: Parto normal; Cesárea; Assistência perinatal; Enfermagem obstétrica.

\section{ABSTRACT}

The research aimed to describe the features of a given population studied, as civil state, occupation and number of pre-natal appointments; to verify the incidence of cesarean section and normal labor in clients attended in an institution; to compare these occurrence in the period of research, discussing the indications of cesarean sections more frequently referred. It is a descriptive-retrospective study based upon patient records from a philanthropic institution of the National Health System. It highlighted a rising percentage of cesarean sections with high incidence in teenagers. It evidenced that the main indications of cesarean section were due to former cesarean section and pregnancy specific hipertensive disease. It was recorded predominance of pregnants with 4 to 7 pre-natal appointments. Changes in pre-natal care can impact in cesarean section rate, preparing the mother to natural labor, something that can be closely related to midwives obstetrical nurses and physicians capacitation, as well as the placement of group care instead individual care.

Descriptors: Natural chidbirth; Cesarean section; Perinatal Care; Obstetrical nursing.

\section{RESUMEN}

La investigación tuvo como objetivo describir las características de la población estudiada, como estado civil, ocupación y número de consultas de prenatal; verificar la incidencia de cesárea y de parto normal en clientes atendidas en una institución; comparar esas incidencias en el período investigado, discutiendo las indicaciones de cesáreas más frecuentemente referidas. Estudio descriptivo retrospectivo basado en prontuarios de una institución filantrópica conveniada al Sistema Único de Salud. Se ha evidenciado un elevado porcentaje de parto por cesárea con una alta incidencia en adolescentes. Se ha constatado que las principales indicaciones de cesárea han tenido una historia de cesárea anterior y de enfermedad hipertensiva específica del embarazo. Se ha registrado un predominio de mujeres embarazadas gestantes con 4 a 7 consultas de prenatal. Cambios en la asistencia prenatal pueden tener impacto en la tasa de cesáreas realizadas, preparando a la madre para el trabajo de parto, aspecto íntimamente relacionado a la capacitación de parteras, enfermeras obstetras y médicos, como también a la sustitución de la asistencia individual por una asistencia de equipo.

Descriptores: Parto normal; Cesárea; Atención perinatal; Enfermería obstétrica.

Queiroz MVO, Jacobina e Silva NS, Jorge MSB, Moreira TMM. Incidência e características de Cesáreas e de partos normais: estudo em uma cidade no interior do Ceará. Rev Bras Enferm 2005 nov-dez; 58(6):687-91.

\section{INTRODUÇÃO}

Nas últimas décadas tem ocorrido em todo o mundo uma crescente incidência de operações cesarianas. O Brasil tem lugar de destaque nesse cenário, pois apresenta uma das maiores taxas do mundo. Entre os anos de 1991 a 1995, a taxa média de cesariana se elevou de 22\% para 27\%. Este aumento também é registrado em vários países latino-americanos ${ }^{(1)}$. 
Quando se analisa a incidência de cesarianas em relação ao nível socioeconômico, vê-se o paradoxo de que, à medida que aumenta a renda média da população estudada (e, por conseguinte, diminui o risco médico desta), aumenta também a incidência do parto operatório.

A cesariana é uma intervenção cirúrgica que possibilita que o bebê seja retirado do útero materno, em vez de nascer naturalmente, passando pelo colo do útero e vagina. Esta intervenção é definida como o nascimento do feto mediante incisão nas paredes abdominal (laparotomia) e uterina (histerotomia) ${ }^{(2)}$.

São vários os fatores que podem indicar a necessidade de um parto por cesariana, tais como apresentações anômalas como apresentação pélvica, crônica ou quando a mãe apresenta doença sexualmente transmissível, como a Síndrome da Imunodeficiência Adquirida (AIDS) e alguns casos de Papilomavírus Humano (HPV), ou ainda, outros motivos, a exemplo do descolamento prematuro da placenta, placenta prévia, diabetes gestacional e Hellp síndrome, dentre outros, que venham a complicar o bom andamento do parto.

Assim, a cesárea é um recurso que permite realizar o parto de maneira satisfatória, quando a vida da mãe ou da criança esteja correndo algum risco. Nesse sentido, a cesariana é um ótimo recurso disponível para proteger, ao mesmo tempo, a saúde da mãe e da criança, permitindo um parto sem maiores complicações ${ }^{(3)}$.

O parto normal é o método natural de nascer e, como tal, possui a proteção das forças da natureza. Se a mãe for jogada à própria sorte, em mais de $92 \%$ das vezes ela terá o seu filho sem problemas. Asua recuperação é imediata, pois, logo após o nascimento, poderá levantar-se e atender seu filho. As complicações próprias do parto normal são menos graves quando comparadas com aquelas advindas do parto cirúrgico. A amamentação do recém-nascido se torna mais fácil e, mais saudável a ele; a infecção hospitalar é muito menos freqüente no parto normal(4). Por outro lado, este produz, pela espera, ansiedade na futura mãe. Esta ansiedade é aumentada também pela preocupação com as dores do parto.

Durante o pré-natal, é ponto fundamental que essa mulher receba orientação sobre formas opcionais para controlar a dor durante o trabalho de parto e que não há justificativa para se realizar uma cesariana apenas com esta finalidade ${ }^{(4)}$.

Com relação às cesarianas, que deveriam ser indicadas apenas nas situações de risco, como citado anteriormente, há hoje um verdadeiro abuso. É importante lembrar que se trata de uma cirurgia que não é das mais simples, com todos os riscos que envolvem qualquer procedimento cirúrgico que implica uso de anestesia, cortes e medicamentos. Obrigatoriamente é realizada em hospitais, o que eleva os riscos de infecção hospitalar para a mãe e o filho. Há um período pós-cirúrgico em que ocorrem dores e desconforto. Assim, se as mulheres pretendem fugir de dores e desconforto, podem estar correndo na direção errada ao optarem pela cesárea sem a devida necessidade.

A vivência de uma das autoras na área obstétrica e a experiência das docentes no ensino e na assistência com o referido tema vem despertar para a realização do estudo, o qual se apresenta de extrema relevância social no campo da atenção à mulher e à criança. Mediante o exposto, desenvolvemos a pesquisa de campo a partir dos objetivos: descrever as características da população estudada, como estado civil, ocupação e número de consultas de pré-natal; verificar a incidência de cesárea e parto normal em mulheres atendidas em uma instituição; comparar essas incidências no período pesquisado, discutindo as indicações de cesarianas mais freqüentemente referidas.

\section{PROCEDIMENTOS METODOLÓGICOS}

O estudo foi do tipo descritivo, retrospectivo e documental, a partir da informação de prontuários. O desenvolvimento deste trabalho se deu em uma instituição filantrópica conveniada ao Sistema Único de Saúde (SUS) da microrregião do maciço de Baturité-Ceará. A unidade se caracteriza como um hospital de referência para essa região na área de ginecologia e obstetrícia, em nível primário e secundário, pois possui berçário de médio risco, atendendo oito municípios.

A população-alvo do estudo constituiu-se de gestantes atendidas nesta instituição filantrópica, compreendidas na faixa etária acima de 14 anos, no período de janeiro de 2003 a janeiro de 2004, totalizando 1.200 prontuários. A amostra tomou como critério de inclusão as gestantes atendidas no turno da manhã, totalizando 65 mulheres $(5,4 \%$ do total) que realizaram o parto no referido hospital e no tempo indicado há pouco.

Os dados coletados na pesquisa em prontuários foram distribuídos estatisticamente sob forma de tabelas simples, com freqüência absoluta e percentual, sendo analisados com base no referencial teórico estudado.

No tocante aos aspectos éticos, o trabalho foi encaminhado à instituição, acompanhado de ofício e do termo de fiel depositário solicitando a entrada no campo de pesquisa, a qual foi concedida, em documento, por seu dirigente. Esse processo se deu após a aprovação pelo Comitê de Ética da Universidade Estadual do Ceará.

\section{APRESENTAÇÃO E INTERPRETAÇÃO DOS DADOS}

Constatamos que de janeiro 2003 a janeiro 2004, ocorreram 1.200 partos na instituição estudada, verificando-se um percentual de 40,0\% de cesáreas. Vejamos o número de cesáreas dentre a amostra estudada (65 partos) na Tabela 1.

Tabela 1. Participantes segundo tipo de parto. Baturité-CE, Janl 2003-Jan/2004.

\begin{tabular}{lcc}
\hline Tipo de Parto & $\mathbf{n}$ & $\%$ \\
\hline Cesárea & 26 & 40,0 \\
Vaginal espontâneo & 39 & 60,0 \\
Vaginal fórceps & - & - \\
\hline Total & 65 & 100,0 \\
\hline
\end{tabular}

Os dados acima evidenciam que a incidência de cesárea continua muito alta, representado em 40,0\%. Tal resultado vai de encontro à orientação da Organização Mundial de Saúde, que recomenda uma taxa de cesárea em torno de $15,0 \%{ }^{(5)}$. No Brasil houve diminuição das clientes do SUS às custas de políticas do Ministério da Saúde em penalizar hospitais públicos que apresentassem taxas superiores a 30,0\%. Deve-se concluir que 15,0\% deve ser a taxa mínima e não a máxima, que ainda não foi estabelecida, o que provavelmente deverá ser algo entre 20,0 a 25,0\%(6).

Relativamente à evolução da incidência de cesárea, percebe-se que é uma cultura mundial a adoção deste tipo de parto, acentuando-se mais nas últimas décadas, atingindo valores altos em determinadas regiões/país mais evoluídos.

Barros, Vougham e Victora( ${ }^{(7)}$, estudando a assistência pré-natal e ao parto em Pelotas (RS), concluíram que os médicos concentravam seus esforços nos clientes de baixo risco e de nível social mais elevado, destacando a incidência de $50,0 \%$ de cesáreas nos clientes particulares e 13,0\% nas mulheres sem seguro de saúde. Inferimos que 0 alto índice de cesáreas para os atendimentos particulares pode estar relacionado com a rápida resolubilidade do processo do parto, assim como a preferência das mulheres por evitar desconfortos próprios do parto normal.

Estudo realizado no Município de Ribeirão Preto (SP), no período de 1986 a 1995, mostra que houve aumento na incidência de cesárea nos atendimentos particulares (68,3\% em 1986 e $81,8 \%$ em 1995) e por planos de saúde ou convênios (69,1\% em 1986 e 77,9\% em 1995), porém ocorreu diminuição nos atendimentos pelo SUS (38,7\% em 1986 e 32,1\% em 1995). Os autores concluíram que a incidência de cesárea aumentou à medida que se elevou o padrão social das gestantes, não havendo correspondência com o risco obstétrico ${ }^{(8)}$. Estudo semelhante realizado em hospitais em São José do Rio Preto (SP), foi encontrado 
um percentual de 93,4\% de cesáreas nos atendimentos por convênio ou particular, enquanto nos atendimentos pelo SUS a proporção foi de $68,3 \%\left({ }^{(9)}\right.$.

Na prática percebe-se que a preferência dos médicos pela cesariana pode ter várias origens. $\mathrm{O}$ que predomina, contudo, é a conveniência de uma intervenção programada, que não tomará mais do que uma hora do seu tempo, ao contrário do parto vaginal, que pode ocorrer a qualquer hora do dia ou noite, e que ocupará um período maior e imprevisível de seu tempo.

Tabela 2. Participantes segundo faixa etária e estado civil. BaturitéCE, Jan/2003-Jan/2004.

\begin{tabular}{clcccc}
\hline Estado Civil & \multicolumn{2}{c}{ Casada } & \multicolumn{2}{c}{ Solteira } & Total \\
\cline { 2 - 5 } Faixa Etária & $\mathbf{N}^{\circ}$ & $\%$ & $\mathbf{N}^{\circ}$ & $\%$ & $(\%)$ \\
\hline $14-18$ anos & 1 & 1,5 & 13 & 20,0 & 21,5 \\
$19-23$ anos & 6 & 9,2 & 15 & 23,0 & 32,2 \\
$24-27$ anos & 6 & 9,2 & 4 & 6,1 & 15,3 \\
$28-32$ anos & 3 & 7,6 & 5 & 7,6 & 15,2 \\
$33-37$ anos & - & - & 5 & 7,6 & 7,6 \\
+37 anos & 5 & 7,6 & - & - & 7,6 \\
\hline Total & $\mathbf{2 3}$ & $\mathbf{3 7 , 0}$ & $\mathbf{4 2}$ & $\mathbf{6 3 , 0}$ & $\mathbf{1 0 0 , 0}$ \\
\hline
\end{tabular}

A tabela 2 evidencia que, em relação à idade materna, a maioria encontrava-se na faixa etária de 19 a 23 anos, representando $32,2 \%$ da amostra na população estudada, chamando a atenção 0 alto percentual de mulheres submetidas ao parto abdominal com idade inferior a 20 anos, verificando-se um elevado percentual de cesáreas entre as adolescentes (21,5\%). Estima-se que, no Brasil, um milhão de adolescentes dão à luz a cada ano, o que corresponde a $20 \%$ do total de nascidos vivos. Estas estatísticas comprovam que em todo o mundo, a cada década, cresce o número de partos de meninos cada vez mais jovens ${ }^{(10)}$.

O fato sugere que as adolescentes estão iniciando a vida sexual mais cedo, apontando para a necessidade de oferta de informação e orientação sobre sexualidade e planejamento familiar para essa parcela da população(11).

Quanto ao estado civil, evidenciado na tabela III, verificamos que a maioria das mulheres era de solteiras na faixa etária 19 a 23 anos, com percentual de 23,0\%. Em 1993, um estudo realizado por Peacek et al., mostrou que, as mulheres adolescentes não brancas e não casadas tinham menor probabilidade de ser submetidas à cesárea do que aquelas de 35 anos ou mais, casadas e brancas ${ }^{(12)}$. Este resultado é esperado em relação à idade tendo em vista que a cesárea poderá ser indicada em mulheres mais velhas para indicação de ligação tubárica, embora a mulher jovem tenha outras indicações. Quanto ao estado civil, fatores psicológicos e preocupações com o futuro podem induzir a decisão de uma cesária.

Tabela 3. Participantes segundo a ocupação. Baturité-CE, Jan/2003Jan/2004.

\begin{tabular}{lcc}
\hline Ocupação & $\mathbf{n}$ & $\%$ \\
\hline Agricultura & 47 & 72,3 \\
Doméstica & 2 & 3,0 \\
Costureira & 1 & 1,5 \\
Estudante & 4 & 6,1 \\
Sem ocupação & 4 & 6,1 \\
Auxiliar de escritório & 1 & 1,5 \\
Professora & 1 & 1,5 \\
Do lar & 4 & 6,1 \\
Auxiliar de enfermagem & 1 & 1,5 \\
\hline Total & $\mathbf{6 5}$ & $\mathbf{1 0 0 , 0}$ \\
\hline
\end{tabular}

A tabela 3 mostra um elevado número de mulheres sem trabalho remunerado, sendo $72,3 \%$ são agricultoras, vivendo do que produzem no campo.

Essa realidade socioeconômica parece preocupante para a saúde desta mulher e de seu filho, pois conforme 0 Ministério da Saúde ${ }^{(13)}$, a mulher de baixa renda carece de alimentação, moradia, trabalho, educação, cultura, lazer, entre outras necessidades. Evidentemente, essa "doença social" determina ou agrava as condições biológicas e psicológicas que lhe põem em risco a sua vida, a sua saúde e o seu bem-estar.

Tabela 4. Participantes segundo o número de consultas de prénatal. Baturité-CE, Jan/2003-Jan/2004.

\begin{tabular}{lcc}
\hline Consultas de Pré-Natal & $\mathbf{n}$ & $\%$ \\
\hline Nenhuma & 3 & 4,7 \\
1 a 3 & 8 & 12,3 \\
4 a 6 & 26 & 40,0 \\
+ de 6 & 28 & 43,0 \\
\hline Total & 65 & $\mathbf{1 0 0 , 0}$ \\
\hline
\end{tabular}

Conforme a tabela 4, a maioria das mulheres realizou entre quatro e sete consultas de pré-natal (83\%), enquanto apenas 17\% tiveram até três consultas. Esse número parece-nos próximo do preconizado pelo Ministério da Saúde, que recomenda seis consultas para um pré-natal de qualidade.

O pré-natal é o momento mais apropriado para a preparação e orientação para o parto e detecção de possíveis intercorrências. A literatura demonstra que a renda familiar, a escolaridade materna, a situação conjugal, o local de residência e a paridade da mulher estão associados à utilização dos serviços de saúde, incluindo-se assistência pré-natal e hospitalar ao parto Para o Ministério da Saúde, é considerada realizada a conclusão da assistência pré-natal quando tiverem sido realizadas seis consultas de pré-natal e todos os exames obrigatórios, devendo-se iniciar o mais precocemente possível ${ }^{(13)}$.

Um estudo realizado em Fortaleza, nos anos 1995 a 1996, mostrou elevada cobertura pré-natal, abrangendo $92 \%$ da população-alvo, porém, em $30 \%$ das mulheres entrevistadas, a assistência foi considerada inadequada com início tardio e/ou número de consultas insuficientes. Dentre os obstáculos citados para a não-realização do pré-natal adequado, destacaram-se a dificuldade de acesso ao serviço e a má qualidade do atendimento ${ }^{(11)}$.

Tabela 5. Participantes segundo a indicação para a cesárea mais freqüentemente referida. Baturité-CE, Jan/2003-Jan/2004.

\begin{tabular}{lcc}
\hline Indicação de Cesárea & $\mathbf{n}$ & $\%$ \\
\hline Cesárea anterior & 7 & 27,0 \\
DHEG & 5 & 19,2 \\
DCP & 5 & 19,2 \\
Amniorrexe & 3 & 11,5 \\
Apresentação anômala & 4 & 15,4 \\
Outras & 2 & 7,7 \\
\hline Total & 65 & 100,0 \\
\hline
\end{tabular}

A tabela 5 mostra que a principal indicação do parto abdominal foi a cesárea anterior (27,0\%), sendo a DHEG responsável por 19,2\% e DCP $19,2 \%$. Na categoria outros, estão inclusos os casos de generalidade, prolapso de cordão, malogro de indução, deslocamento prematuro da placenta (DPP), sofrimento fetal agudo (SFA), placenta prévia, dentre outros.

No Brasil, estudiosos do assunto encontraram a DCP como principal indicação de cesárea $(39,2 \%)$, seguindo-se a cesárea iterativa ou cesárea anterior $(16,9 \%)$, a apresentação pélvica $(8,0 \%)$ e a doença hipertensiva $(5,7 \%)^{(14)}$. O estudo de Yazlle et al. realizado em Ribeirão Preto (SP), mostrou que as principais indicações referidas foram 0 sofrimento fetal e a distócia cefalo-pélvica ${ }^{(8)}$.

É provável que alguns motivos da indicação da cesárea descritos no prontuário não correspondam à realidade. $A$ análise de prontuários de mulheres que pariram numa maternidade no Rio de Janeiro revelou que, em 54 cesáreas realizadas, em treze houve indicação incorreta e, em outras treze, a indicação era questionável(15). 
Tabela 6. Participantes segundo a incidência de partos normais e partos cesáreas por faixa etária. Baturité-CE, Jan/2003-Jan/2004.

\begin{tabular}{ccccccc}
\hline Tipos de Parto & \multicolumn{2}{c}{ Partos Normais } & \multicolumn{2}{c}{ Partos Cesáreas } & \multicolumn{2}{c}{ Total } \\
\cline { 2 - 7 } Faixa Etária & $\mathbf{n}$ & $\%$ & $\mathbf{n}$ & $\%$ & $\mathbf{n}$ & $\%$ \\
\hline $14-18$ anos & 6 & 9,2 & 8 & 12,3 & 14 & 21,5 \\
$19-23$ anos & 13 & 20,0 & 8 & 12,3 & 21 & 32,3 \\
$24-27$ anos & 5 & 7,6 & 6 & 9,3 & 11 & 17,0 \\
$28-32$ anos & 6 & 9,2 & 4 & 6,1 & 10 & 15,3 \\
$33-37$ anos & 4 & 6,1 & - & - & 4 & 6,1 \\
+37 anos & 5 & 7,6 & - & - & 5 & 7,6 \\
\hline Total & $\mathbf{3 9}$ & $\mathbf{5 9 , 7}$ & $\mathbf{2 6}$ & $\mathbf{4 0 , 0}$ & $\mathbf{6 5}$ & $\mathbf{1 0 0 , 0}$ \\
\hline
\end{tabular}

A tabela 6 evidencia a relação entre parto normal e parto cesárea com relação à faixa etária, e chama a atenção para o fato de que 0 percentual de cesárea é maior entre as adolescentes na faixa etária de 14 a 18 anos, correspondendo a 12, 3\%, quando parto normal na mesma faixa etária foi de $9,2 \%$. Isto é muito preocupante, pois, como no Brasil a prática é depois de uma cesárea sempre cesárea, essa mulher terá limitado a sua prole, e muitas vezes, por uma primeira cesárea, nem sempre bem indicada, sofrerá essa intervenção nas gestações seguintes, o que também onera os serviços de saúde e eleva índices de morbimortalidade.

A cicatriz uterina pode ter menos influência sobre a evolução do parto do que inicialmente se pensava, porém aumenta o risco de algumas sérias complicações, como placenta prévia, outras patologias hemorrágicas, morbidade neonatal e infecções puerperais ${ }^{(16)}$.

Um efeito final da alta prevalência de cesáreas é a limitação da fecundidade entre a população envolvida. Em circunstâncias de taxas excepcionalmente altas, como no Brasil, e, sobretudo, quando somente os casos rápidos e fáceis evoluem para parto vaginal, a incidência entre nulíparas deve ser ainda mais altos. Como a prática geral é, depois de uma cesárea, sempre cesáreas, e a esterilização realizada sistematicamente após a terceira cesárea, todas essas mulheres terão no máximo três filhos ${ }^{(17)}$.

Para o médico, a decisão de realizar uma cesárea eletiva, no lugar de um parto vaginal, pode parecer não ter nenhuma conseqüência econômica. Asoma de muitas decisões semelhantes, contudo, tomadas diariamente, pode afetar seriamente o custo da assistência a saúde materno-infantil. No hospital da UNICAMP, a estimativa da diferença de custo entre os dois tipos de parto foi de $50 \%$ maior para a cesárea ${ }^{(17)}$. Esse desperdício de recursos já escassos é condenável, em razão das deficiências na assistência pré-natal básica, dos baixos índices de diagnósticos preventivos e das deficiências em várias outras necessidades básicas de saúde ${ }^{(18)}$

Diante de todas as observações feitas, vê-se claramente que o custo da operação cesariana é muito alto em comparação com o parto normal.
Na cesárea há cortes, gastos com material e medicamentos, risco de infecção e, ainda, o risco de se fazer um parto antes do tempo, o que eleva ainda mais os gastos com um recém-nascido prematuro que necessitaria de cuidados especiais. Para o Ministério da Saúde, um mecanismo que aumenta 0 curso da assistência à saúde é o derivado da prematuridade iatrogênica. Um bebê prematuro requer uma assistência neonatal intensiva por algum tempo, o que aumentará a demanda e os custos dos serviços de saúde ${ }^{(13)}$. Já o parto normal ocorre de modo natural, com um mínimo de intervenção e, logo após dar à luz, essa mulher poderá levantar-se, cuidar de seu filho, amamentá-lo sem dores ou desconfortos, que são próprios do parto cesáreo.

\section{CONSIDERAÇÕES FINAIS}

Mediante a proposta e com base nos achados conclui-se que o índice de cesáreas é elevado na instituição pesquisada 40,0\% (amostra representativa), refletindo a tendência observada no Brasil e no mundo. Tal realidade leva-nos a supor que as indicações de cesariana não estão sendo precisas, denotadas pela indicação nem sempre clara nos prontuários de se realizar o parto abdominal, seja pela conveniência médica, seja por treinamento obstétrico incompleto, que deixaria 0 médico inseguro e qualquer distorcia mínima real ou imaginária resultaria na decisão de se realizar uma cesárea; um elevado percentual de mulheres que se submeteu à cesárea encontra-se na faixa etária de 19 a 23 anos, verificando-se também, uma alta proporção de adolescentes (21,5\%); constatou-se que a maioria não tinha companheiro, e não tinha trabalho remunerado $(72,3 \%$ eram agricultoras), ou seja, pertenciam a classe social menos favorecida.

É interessante salientar que as principais indicações de cesárea foram a cesárea anterior (27,0\%), seguida da DHCG $(19,2 \%)$, DCP $(19,2 \%)$, amniorrexe $(11,5 \%)$, apresentação anômala $(15,4 \%)$ e outros $(7,1 \%)$. Houve predomínio de gestantes com 4 a 7 consultas de prénatal que deveria preparar psicologicamente essa mulher para o parto, principalmente, esclarecendo as vantagens do parto normal, bem como detectar precocemente complicações e prevenindo as principais afecções perinatais. Nota-se que essas mulheres realizam um número de consultas de pré-natal recomendado pelo Ministério da Saúde, porém esse pré-natal ainda não é de qualidade, visto que as mulheres, ao chegarem ao hospital estão despreparadas para "dar à luz", acreditando que a dor fisiológica do parto é sofrimento e que a cesárea as livraria de todos esses incômodos.

Finalmente, acredita-se que mudanças na assistência pré-natal podem, também, ter um impacto na taxa de cesárea, preparando a mãe para o trabalho de parto e parto. Esse aspecto está relacionado intimamente com o resgate da profissão da parteira, ao treinamento dos médicos e outros profissionais de saúde, como a enfermeira obstetra, e a substituição da assistência individual por uma que envolva a equipe.

\section{REFERÊNCIAS}

1. CLAP. Salud perinatal. Bol Clap 1989; 3: 9 .

2. Freitas F. Rotinas em obstetrícia. $4^{a}$ ed. Porto Alegre (rs): Artmed; 2001.

3. Ministério da Saúde (BR). Parto, aborto e puerpério: assistência humanizada à mulher. Brasília (DF): MS; 2001.

4. Parto normal $X$ cesárea. [citado em: 24 abr 2004]. Disponível em: URL: http://www.dremar.med. br/PartoCesarea.htm

5. World Health Organization (WHO). Appropriate technology for birth Lancet 1995; 24: 436-7.

6. Jornal da Febrasgo. Parto $X$ cesárea: situação atual no Brasil. J Febrasgo 2001; 4(1): 2-6.

7. Barros FC, Vaugham JP, Victora CG. Why so many cesarean sections? The need for a further policy change in Brasil. Health Police Plan,1986.

8. Iazlle MEHD. Incidência de cesáreas segundo fonte de financiamento da assistência ao parto. Rev Saúde Pub 2001; 35(2): 202-6.

9. Moraes MS, Goldenberg P. Cesáreas: um perfil epidêmico. Cad. Saúde Pub 2001; 17(3): 509-19.

10. Ramos FRS, Monticelli M, Nitschke RG organizadores. Projeto acolher: um encontro de enfermagem com 0 adolescente brasileiro. Brasília (DF): ABEn/Governo Federal; 2000.

11. Secretaria de Saúde do Estado do Ceará (CE). Agência de Cooperação Internacional do Japão (JICA). Fortaleza (CE); 1998.

12. Shearer EL. Cesarean section: medical benefits and costs. Soc Sci Med 1993; 37(16): 1223-31.

13. Ministério da Saúde (BR). Parto, aborto e puerpério: assistência humanizada à mulher. Brasília (DF): MS, 2000. 
Incidência e características de Cesáreas e de partos normais: estudo em uma cidade no interior do Ceará

14. Cunha AA, Portela MC, Amed AM, Camano L. Incidência de cesárea - um problema? Femina 2001; 28(10): 553-6.

15. Dias M. Medidas para redução da taxa de cesariana. In: Anais do $1^{\circ}$ Relatório de Conferência Nacional sobre Organização de Serviços para Maternidade Segura à Luz da Humanização - Projeto Luz; 1998.

16. Moraguez AJD, Silva JLP, Pinotti J.A., Faúndes A. Prognóstico obstétrico e perinatal da mulher portadora de cicatriz de operação cesariana. Gin Obst Bras 1991; 4:173.

17. Faúndes A, Silva JLP. O equilíbrio entre o parto vaginal e a cesárea na assistência obstétrica atual. Femina 1998; 26(8): 669-673.

18. Rocha JSY, Ortez PC, Fung, YT. A incidência da cesárea e a remuneração da assistência ao parto. Rev Paul Med 1996; 104:167. 